\title{
Covering the Target Objects with Mobile Sensors by Using Genetic Algorithm in Wireless Sensor Networks
}

\author{
Van-Dai Ta1 ${ }^{*}$, Shih-Chang Huang1, Huynh Thi Thanh Binh ${ }^{2}$ \\ ${ }^{1}$ Department of Computer Science and Information Engineering, National Formosa University, Yunlin, \\ Taiwan. \\ ${ }^{2}$ School of Information and Communication Technology, Hanoi University of Science and Technology, \\ Vietnam. \\ * Corresponding author. Email: daitv88@gmail.com \\ Manuscript submitted March 20, 2015; accepted July 10, 2015. \\ doi: $10.17706 /$ jcp.10.5.300-308
}

\begin{abstract}
Wireless sensor networks have several applications, such as target detection and tracking, and monitoring battlefields. Coverage is one of the most important performance metrics for wireless sensor networks since it reflects how appropriate an event can be detected and monitored in the sensing field. To achieve optimal coverage, an efficient algorithm should be employed to find the best positions of sensor node deployment. In this paper, an efficient genetic algorithm is proposed to solve the coverage problem of the target objects. The optimal number of sensor nodes starts from fewer randomly deployed nodes and increases gradually in subsequence generations. The performance of the proposed genetic algorithm was evaluated, and the simulation results show that this approach can cover all the target objects as well as minimize the number of additional mobile sensor nodes.
\end{abstract}

Key words: Wireless sensor networks, sensor deployment, target object coverage, genetic algorithm.

\section{Introduction}

Over the past decade, there has been a considerable increase in research, development, and integration of wireless sensor networks (WSNs), introducing a wide variety of exciting new applications, and recently becoming an integral part in the Internet of Things (IoT) concept [1].

The deployment strategy is the prerequisite in the WSNs. It affects almost all its performance metrics, such as the connectivity between sensors, and the effective coverage and lifetime of networks. In general, WSN deployment methods fall under two categories: deterministic deployment and random deployment [2]. Deterministic is a method which defines the locations of the sensor nodes in advance to maximize coverage, minimize power consumption, or achieve strong network connectivity. Random deployment is the only feasible solution in some applications to monitor hostile environments, such as wild fires, disaster areas, and active war zones where static sensor deployment cannot be performed manually. Logically, random deployments result in sub-optimal performance of the WSNs. However, the disadvantage of random deployment is the unbalanced density of the sensors on monitored areas which can cause the holes problem in WSNs [3]. Therefore, in most cases, random deployment is not ensured to meet the required objective in terms of the coverage.

Coverage is one of the fundamental issue and an active research area in WSNs. It is the measure of the Quality of Service (QoS) that is how well the targets are being covered by the deployed set of sensors. The 
aim of coverage is that each object in the physical space of interest must be within sensing range of at least one sensor. Coverage problem has been the subject of various studies. The coverage problem is grouped into three categories: area coverage, where the goal is to have each point of the region be within a detection distance from at least one of the sensors; barrier coverage, where the objective is to optimally protect the region from undetected penetration; target coverage, where the goal is to move a number of sensors across the region as to maximize the probability of detecting a target.

Optimal sensor placement focuses on maximizing coverage and minimizing the number of sensor nodes. It is a type of the minimum set cover problem, which can be proven to be non-deterministic polynomial time (NP-hard) [4]. A genetic algorithm is a good approach for this kind of optimization. The maximum coverage approach ensures that all the target objects in the given area are covered by the optimal number of sensor nodes. We define the problem formally and propose an efficient genetic algorithm (GA) to improve the optimal coverage of the network. Minimum number of additional mobile sensors is added to static node set initialized with a small number of nodes. Many target objects are both randomly and uniformly distributed to evaluate the efficiency of GA.

The rest of this paper is organized as follows: Section 2 presents the target object coverage deployment, Section 3 introduces the related works, and Section 4 presents the proposed GA for sensor deployment to improve the coverage of WSNs. The discussions of the experiment results are given in Section 5, and Section 6 provides the conclusion of the paper.

\section{Target Object Coverage Deployment}

\subsection{Assumptions}

- During the initial step, the static nodes are randomly deployed in the sensing field. Sensor nodes have a GPS component to retrieve their locations.

- We assumed that each sensor node with a sensing radius $r$ can cover a circular area. A target object is covered by a sensor if it is within the sensing area of the sensor. This can be represented by the Boolean disk coverage model [5]. The coverage function of the model is given by

$$
f\left(d\left(S_{i}, O_{j}\right)\right)= \begin{cases}1, & \text { if } d\left(S_{i}, O_{j}\right) \leq r \\ 0, & \text { Otherwise }\end{cases}
$$

In (1), $d\left(S_{i}, O_{j}\right)$ is the Euclidean distance between the location of sensor $S_{i}$ and the object $O_{j}$.It is implied that all objects within the circle are covered by this sensor. So, the coverage function returns 1 . On the contrary, the coverage function returns 0 if the uncovered objects are outside the circle.

- Few mobile nodes are used to cover the rest of the target objects, which are not covered by the initial deployed static nodes. Furthermore, new positions of the newly added mobile sensor nodes are determined by the base station node. The location of the base station node is at the center of the sensing field. The optimal number of sensor nodes is also selected by the base station node.

\subsection{Problem Formulation}

We define the maximum coverage problem of sensor deployment in the following way. There are $N$ sensor nodes with the same sensing range $r$ which are randomly deployed in field $A$. $S=\left\{S_{1}, S_{2}, \ldots, S_{i}, \ldots S_{N}\right\}(i=1$ to $N)$, and $P$ target objects, denoted as $0 . O=\left\{O_{1}, O_{2}, \ldots, O_{j}, \ldots O_{P}\right\}(j=1$ to $P)$

Selected nodes set is represented as Selected Nodes $\operatorname{Set}(k)=\left\{C, H_{k}\right\}$, is a collection of the initial static nodes and additional mobile nodes that can improve the coverage of network. Selected nodes set gets the optimal value after $k$ iterations, when all the target objects are covered or the limitation iteration $n$ is 
reached.

The initial static nodes $C$ is portioned into two subsets: $C_{1}$ and $C_{2} . C_{1}$ is the subset of nodes that can cover at least one target object. $C_{2}$ is the subset of nodes that does not cover any target objects. The relations of sets $C, C_{1}$, and $C_{2}$ are $C_{1} \cap C_{2}=\emptyset$ and $C_{1} \cup C_{2}=C . M_{i}$ is the set of mobile sensors that are added to the selected nodes set at the $i^{\text {th }}$ iteration. $H_{k}$ is the set of the additional mobile nodes that are added to the selected nodes set after $k$ iterations by evaluating the fitness function.

$$
H_{k}=\bigcup_{i=1}^{k} M_{i}(k \leq n)
$$

Our objective is to minimize the number of additional mobile nodes as well as coverall the target objects.

$$
\text { Objective: Minimize }\left|H_{k}\right|
$$

\section{Related Works}

Many studies for efficient sensor placement under varieties of situation and environment have been proposed to achieve maximum coverage in WSNs [5]-[7]. Wang [5] presents a deep survey of coverage problems in sensor networks. The strategies for solving the coverage problem are categorized as the force-based, the grid-based, and the computational geometry based [8]; Force-based deployment strategies are based on the sensors' mobility, using virtual repulsive and attractive forces. The sensors are forced to move away or towards each other so that the full coverage is achieved. There are many studies using the virtual force algorithm (VFA) to solve the coverage problem of WSNs. The advantages of the potential field method are that each sensor does not need any prior information of the environment, and localization.

Grid points are used either to measure coverage as used in VFA or to determine sensors positions when sensors are deployed. Sampling method is commonly used for measuring the degree of coverage. The coverage is estimated as the ratio of covered grid points to the total number of grid points in the ROI [9]. The predetermine deployment method uses grid points as the locations of sensor nodes. This method promises to provide a certain percentage degree of coverage and connectivity [10]. However, the grid size is chosen based on the density of WSNs.

The most commonly used computational geometry approach is Voronoi diagram and Delaunay triangulation. In this approach, the sensor movement is based on the principle of moving sensors from densely deployed areas to sparsely deployed areas. Distributed sensor deployment strategies were proposed in [11] for efficient field coverage in a mobile sensor network. Under these strategies, each sensor moves iteratively in a direction that reduces the coverage holes in its Voronoi polygon. However, the disadvantages of these strategies are that they are centralized thus they may not scale well for larger networks [12].

Recently, many evolution computation approaches were proposed to optimize the deployment and maximize the coverage area of the network. Genetic Algorithm (GA) is one of the most powerful heuristics for solving optimization problems. Basically, GA is based on natural selection and the biological evolution. There have been some studies using GA to solve the coverage problem of optimal node deployment in [13], [14]. In [15] a GA based multiple objectives methodology was implemented for a self-organizing wireless sensor network. In order to maintain the network coverage and energy efficiency, a genetic algorithm was proposed in [16]. Kim and Yoon proposed an efficient genetic algorithm for maximum coverage deployment in WSNs by using heterogeneous sensor nodes [17].

In terms of maximizing coverage deployment and minimizing the number of sensor nodes, this paper proposed a genetic algorithm that finds the minimum number of additional mobile nodes in order to cover all the target objects. 


\section{Proposed Methods}

The main idea of using GA in our model is to find the new locations from the high fitness initial sensor nodes so the mobile sensor nodes can be adopted in those locations. The selection process of GA is based on high fitness to minimize the number of sensor nodes.

\subsection{Individual Representation}

In genetic algorithm, a population of candidate solutions is indicated by the number of individuals. Each individual has a set of properties, which are called chromosomes. These chromosomes can be mutated and altered through the production reproduction process. Traditionally, individuals are represented in binary strings composed of 0 and 1 , but other encodings are also possible. In this paper, each sensor node is an individual, and each individual uses the coordinate of $X$ and $Y$ to represent its chromosomes. For example, the sensor individual located at $(20,14)$ will use 00101000001110 to represent its corresponding chromosomes shown in Fig. 1.

\begin{tabular}{|l|l|l|l|l|l|l|l|l|l|l|l|l|l|} 
X-Location & Y-Location \\
\hline 0 & 0 & 1 & 0 & 1 & 0 & 0 & 0 & 0 & 0 & 1 & 1 & 1 & 0 \\
\hline
\end{tabular}

Fig. 1. Individual chromosome representation.

For example, as illustrated in Fig. 2(a), there are 4 sensor nodes initially deployed in an area to cover 6 target objects. Initial selected nodes set $=\left\{S_{1}, S_{2}, S_{3}, S_{4}\right\}$, here $C_{1}=\left\{S_{1}, S_{2}\right\}$ and $C_{2}=\left\{S_{3}, S_{4}\right\}$.

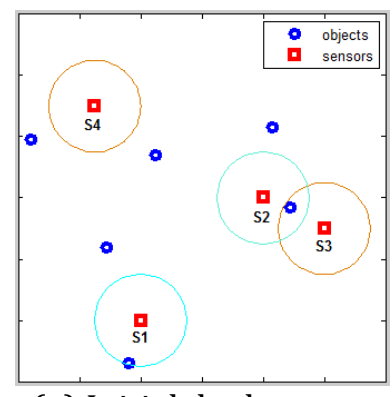

(a) Initial deployment

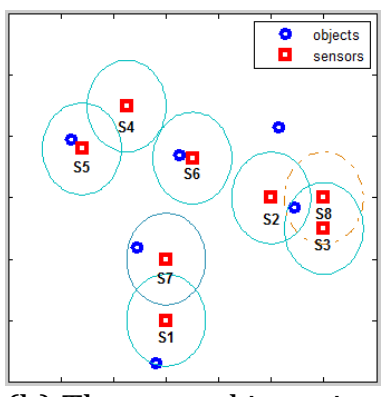

(b) The second iteration

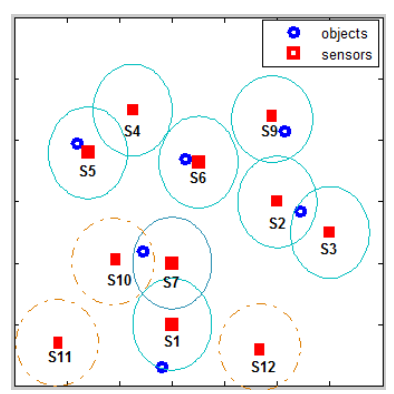

(c) The third iteration

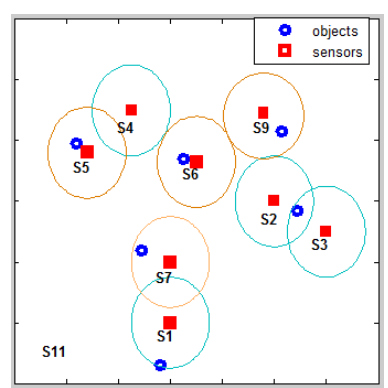

(d) Optimal deployment

\subsection{Fitness Evaluation}

In each generation, the fitness of every individual in the population is evaluated by the fitness function. The fitness function is usually the value of the objective function in the optimization problem being solved. In our model, fitness function calculates the maximum number of covered targets by each sensor node if and only if these targets are uncovered by other mobile or static nodes. This property of fitness function eliminates the overlapping redundancy among the coverage regions of deployed mobile nodes and allows each mobile node to cover only a distinct region. The fitness function to evaluate static and mobile sensor nodes is different.

The fitness of those static sensor nodes in the initial evaluation of population is given in (3). This function calculates the number of covered targets $O_{j}$ by the static node $S_{i}$. The value of $F\left(S_{i}\right)$ will increase by 1 when it covers target object $O_{j}$ that is not covered by any other static nodes.

$$
F\left(S_{i}\right)=\left\{\begin{array}{c}
F\left(S_{i}\right)+1 \text { if } D\left(S_{i}, O_{j}\right) \leq r \text { and } O_{j} \notin\left\{S_{\sim i}\right\} \\
F\left(S_{i}\right), \text { Otherwise }
\end{array}\right.
$$


In Fig. 2(a), the fitness function (3) calculates the number of covered targets of the initial static nodes. $F\left(S_{1}\right)=1 ; F\left(S_{2}\right)=1 ; F\left(S_{3}\right)=0 ; F\left(S_{4}\right)=0$. Although there is one inside target of $S_{3}$ but it is covered by the static node $S_{2}$ thus $F\left(S_{3}\right)=0$. The fitness of initialization is $F(N)=2$.

The fitness of the candidate additional mobile sensor is given in (4). This function calculates the number of covered targets $O_{j}$ by the mobile sensor node $M_{S i}$. The value of $F\left(M_{S i}\right)$ will increase by 1 when it covers target object $O_{j}$ that is not covered by any static nodes and other mobile nodes.

$$
F\left(M_{S i}\right)=\left\{\begin{array}{c}
\left.F\left(M_{S i}\right)+1 \text { if } D\left(S_{i}, O_{j}\right)<r \text { and } O_{j} \notin\left\{S_{\sim i}, M_{S / i}\right)\right\} \\
F\left(M_{S i}\right), \text { Otherwise }
\end{array}\right.
$$

\subsection{Selection}

During each successive generation, a proportion with high fitness of the existing population is selected to breed a new generation. Individual solutions are selected through a fitness-based process by the fitness function.

In this paper, the initial population size will start with a number of initial sensor nodes. Based on the fitness evaluation for the static nodes we can select which nodes are going to be used for crossover and mutation. By evaluating the fitness of newly produced individuals through GA operators, there are some new potential sensor nodes to be added to the selected node set so that we can get the optimal number of additional mobile nodes.

\subsection{GA Operators}

For each new solution to be produced, a pair of parent solutions is selected for breeding from the pool selected previously. A new solution is created, which typically shares many of the characteristics of its parents. New parents are selected for each new child, and the process continues until an appropriately sized new population of solutions is generated.

In this paper, each chromosome pair $(x, y)$ of every sensor is considered as a pair of the parent. A crossover operator that randomly selects a crossover point within a chromosome then interchanges the two parent chromosomes at this point to produce two new offspring as the possible location. For example, one point crossover is performed on a sensor located at $(20,78)$ to get the new location at $(30,68)$ in Fig. 3.

\begin{tabular}{|l|}
\hline Parent 1: $\mathrm{x}=20$ \\
\hline Parent 2: $\mathrm{y}=78$ \\
\hline
\end{tabular}
\begin{tabular}{|l|l|l|l|l|l|l|l|}
\hline & 0 & 1 & 0 & 1 & 0 & 0 \\
\hline & 0 & 0 & 1 & 1 & 1 & 0 \\
\hline
\end{tabular}

Fig. 3. Crossover operator.

While the crossover performs on the high fitness individuals, the mutation performs on the low fitness individuals. Flip bit mutation is used on both $x$ and $y$ chromosomes. The probability of a mutation of a bit is $\frac{1}{l}$, where $l$ is the length of a binary array. This mutation operator takes the chosen genome and inverts the bits as shown in Fig. 4.

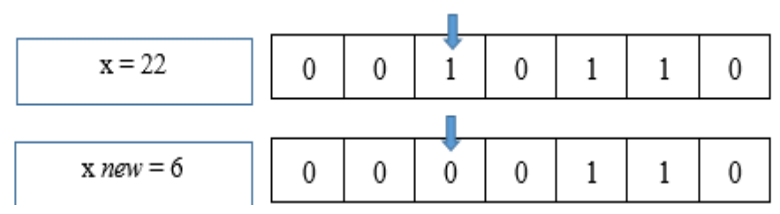

Fig. 4. Mutation operator.

The total fitness $(F)$ is considered as the total coverage of the network at each GA generation. This function is an accumulation the coverage of the static nodes and the added mobile nodes. Total fitness 
function is given by:

$$
F=F(N)+\sum_{i=1}^{m} F\left(M_{S_{i}}\right)
$$

After performing crossover and mutation on $C$, there are 4 new candidate mobile nodes $S_{5}, S_{6}$, $S_{7}, S_{8}$ shown in Fig. 2(b) that are evaluated by the fitness function (4). $F\left(S_{5}\right)=1 ; F\left(S_{6}\right)=1 ; F\left(S_{7}\right)=$ $1 ; F\left(S_{8}\right)=0$. There is one target covered by $S_{8}$ but it is covered by the static node $S_{2}$ so $S_{8}$ is not considered to deploy. The mobile sensor nodes $S_{5}, S_{6}, S_{7}$ are selected for deployment and used for crossover at the next iteration. $S_{8}$ is used for mutation at the next iteration. $M_{1}=\left\{S_{5}, S_{6}, S_{7}\right\}$. In the next iteration, new candidate mobile nodes are shown in Fig. 2(c). $S_{9}$ is added to the selected nodes set, while $S_{10}, S_{11}, S_{12}$ are rejected to deploy. There is one target located inside $S_{10}$ but it is covered by another mobile node $S_{7}$. Therefore, $S_{10}$ is not added to the selected nodes set. $M_{2}=\left\{S_{9}\right\}$. Optimal selected nodes set $=\left\{S_{1}, S_{2}, S_{3}, S_{4}, S_{5}, S_{6}, S_{7}, S_{9}\right\}$. The total fitness is calculated by equation $(5), F=F(N)+F\left(M_{1)}+\right.$ $F\left(M_{2}\right)=6$. The optimal deployment covering all the target objects is presented at Fig. 2(d).

\subsection{Termination}

The generational process repeats until a termination condition is reached. We defined the applied termination condition based on the specific number of generations and coverage ratio perspectives. The algorithm terminates either when the number of iterations is reached or when all the target objects are covered.

Table 1. Experiment parameters 1

\begin{tabular}{ll}
\hline \hline Sensing field area $[\mathrm{m} \times \mathrm{m}]$ & $125 \times 125$ \\
Number of static nodes & 20 \\
Sensing range $(\mathrm{m})$ & 12 \\
Limitation generations & 30 \\
\hline \hline
\end{tabular}

Table 2. Experiment parameters 2

\begin{tabular}{ll}
\hline \hline Sensing field area $[m \times m]$ & $125 \times 125$ \\
Number of target objects & 1764 \\
Number of static nodes & 20 \\
Limitation generation & 30 \\
\hline \hline
\end{tabular}

\section{Experiment Results}

In this section, the performance of the proposed GA is evaluated through two aspects: target object coverage and number of sensor nodes. Target object coverage can be treated as point coverage and area coverage when target objects are deployed randomly and uniformly respectively. The number of sensor nodes starts with a small number of static nodes then selects the additional mobile sensor nodes until all the target objects are covered so that we can minimize the number of sensor nodes. Furthermore, the coverage ratio, k-coverage, and number of additional mobile nodes are measured in different the number of static sensor nodes and various the sensing range. The first experiment parameters are listed in Table 1. The initial placement of sensors follows random deployment with certain sensing range. We consider two kinds of target object distribution: random and uniform distribution. The number of target objects varies from 100 to 700 by random distribution and 100 to 676 by uniform distribution. In the second experiment, the initial placement of sensor nodes starts with a small number when the sensing range varies from 8 to $20 \mathrm{~m}$. There are 1,764 target objects in uniform distribution. In this case, the effect of various sensing ranges on coverage degree of target objects was investigated. The parameters used in the second experiment are given in Table 2.

Fig. 5 shows the number of initial static nodes and additional mobile nodes when the target objects are randomly distributed in Fig. 5(a) and uniformly distributed in Fig. 5(b). In both cases, it is shown that the number of sensor nodes increases as the number of target objects increase. It is because increasing the number of target objects will increase the deploying area to be covered. Therefore, more additional mobile 
nodes need to be added into the network if all target objects need to be covered. As shown, the number of additional mobile nodes in Fig. 5(b) is higher in comparison with in Fig. 5(a). It is because when the targets are uniformly distributed, the coverage problem is considered as the area coverage.

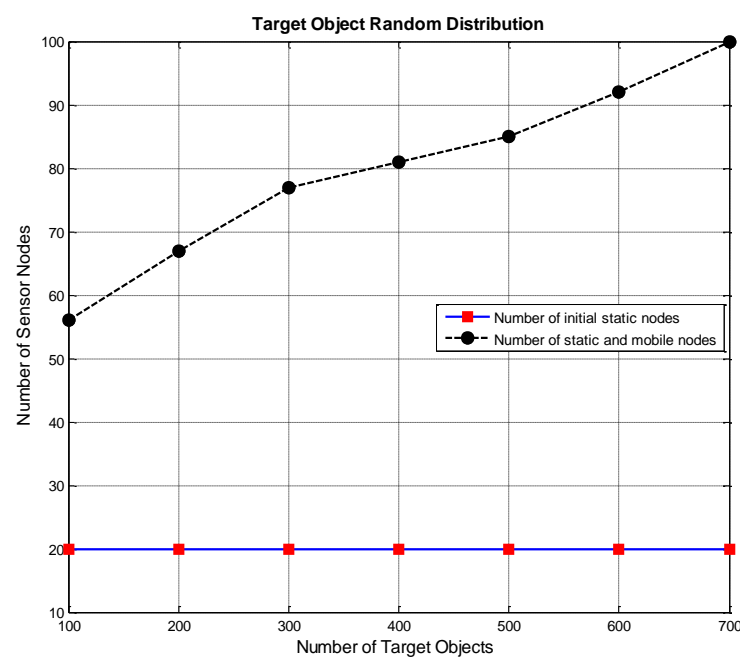

(a)

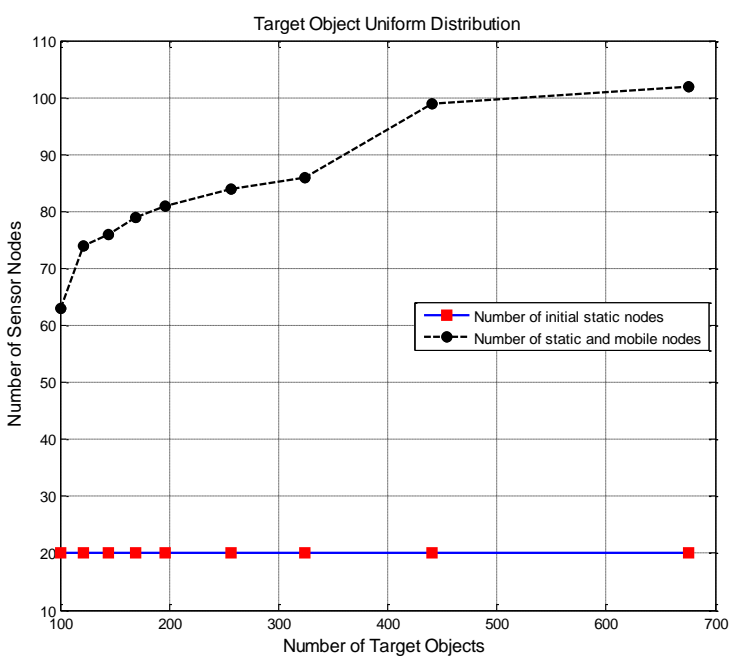

(b)

Fig. 5. Number of sensor node versus target objects distribution.

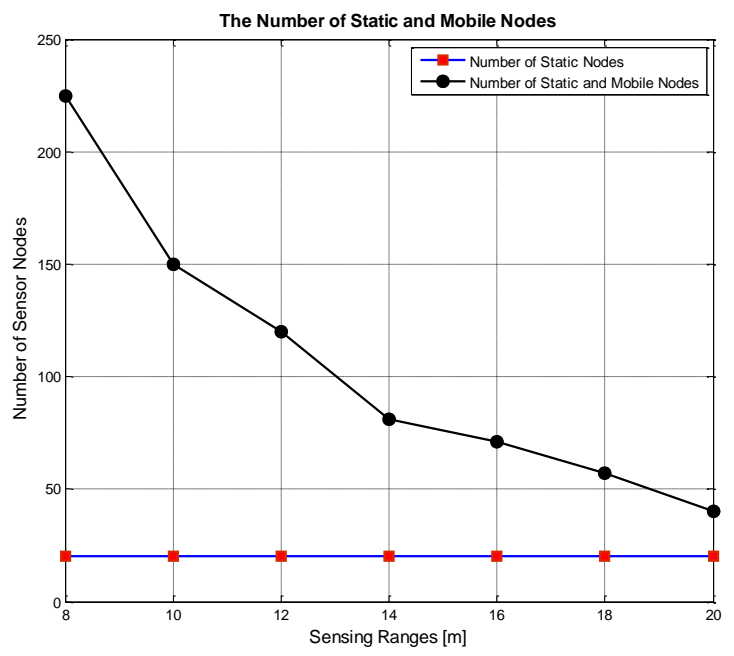

(a)

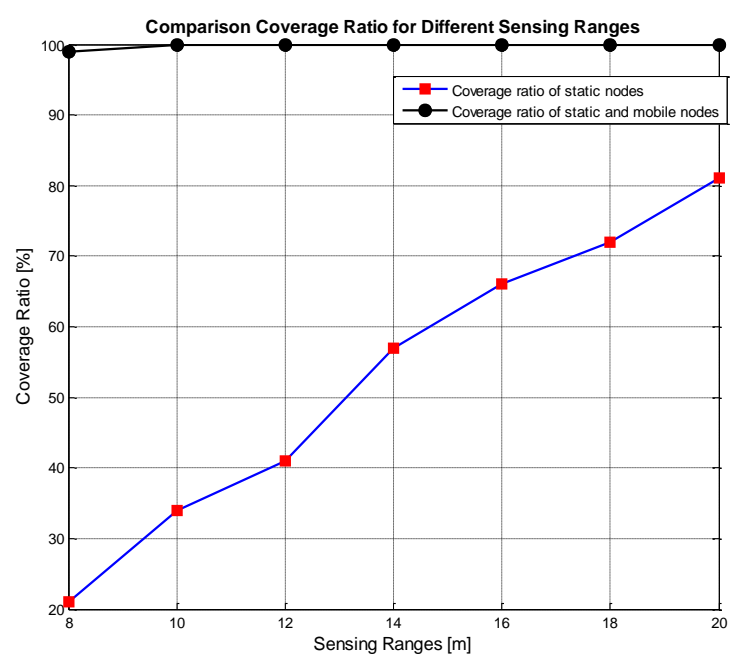

(b)

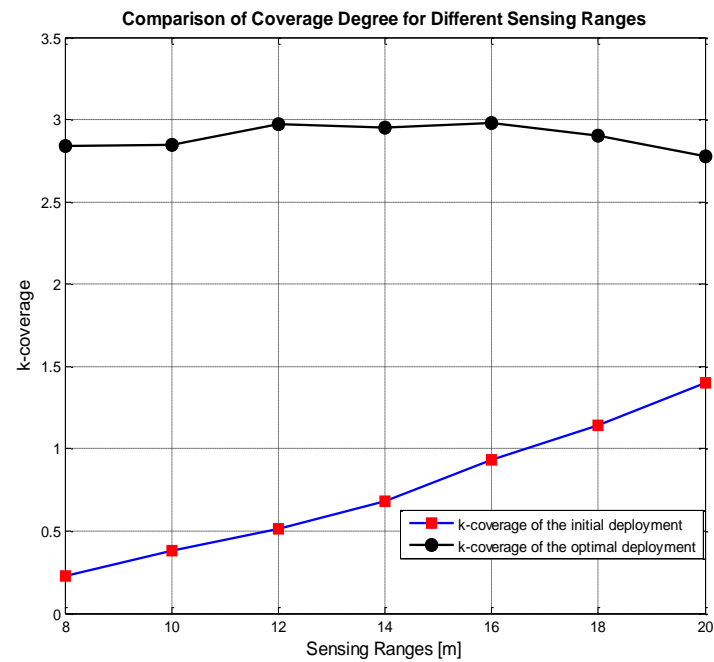

(c)

Fig. 6. Sensor deployment under various sensing ranges. 
Fig. 6 shows the sensor deployment under the various sensing ranges. Fig. 6(a) shows the number of mobile nodes when a small number of static nodes are randomly deployed under various sensing ranges. As shown, the number of sensor nodes decreases as the sensing range increases. This is because more target objects would be covered as the sensing range increases; hence less mobile nodes would be added to cover all the target objects.

Coverage ratio indicates the amount of coverage shown in Fig. 6(b). It is shown that the coverage ratio of the static nodes increases as the sensing range increases. It is due to the fact that sensor nodes with the larger sensing range can cover more target objects than those with smaller range. As shown, the coverage ratio nearly reaches the maximum value after adding the mobile nodes by ensuring all the target objects are covered.

Degree of coverage (k-coverage) which represents every target is covered by $k$ sensors shown in Fig.6(c). As shown, the k-coverage during the initial deployment increases as the sensing range increases. This is because the intersection between sensor nodes increases as the sensing range increases. However, the optimal k-coverage stays relatively stable. This proves the good impact of GA on the high fitness selection of sensor nodes. GA shows the efficiency on reducing the overlapping regions by optimizing the locations of additional mobile sensor nodes and covering every target objects.

\section{Conclusion}

This paper presents a genetic algorithm to find an optimal solution for sensor deployment to cover the number of target objects of both random and uniform distribution. The performance of the proposed algorithm evaluated the number of sensor nodes that were used to cover target objects. In additional, the number of initial static, additional mobile sensor nodes, and k-coverage were measured under various sensing ranges. The simulation results show the genetic algorithm is efficient in target object coverage with high speed computation, optimizing the number of sensor nodes used by minimizing the number of additional mobile sensor nodes in the sensing field. The small number of static nodes in the initial deployment has a good effect on the optimal coverage result and guarantees to cover all the targets.

\section{References}

[1] Mainetti, L., Patrono, L., \& Vilei, A. (2011). Evolution of wireless sensor networks towards the internet of things: A survey. Proceedings of the 19th International Conference on Software, Telecommunications and Computer Networks (SoftCOM) (pp. 1-6). NJ: IEEE.

[2] Deif, D. S., \& Gadallah, Y. (2014). Classification of wireless sensor networks deployment techniques. Communications Surveys \& Tutorials, 16(2), 834-855.

[3] Huang, C. F., \& Tseng, Y. C. (2005). The coverage problem in a wireless sensor network. Mobile Networks and Applications, 10(4), 519-528.

[4] Garey, M. R., \& Johnson, D. S. (2002). Computers and Intractability, 29.

[5] Wang, B. (2011). Coverage problems in sensor networks: A survey. ACM Computing Surveys (CSUR), 43(4), 32.

[6] Chang, R. S., \& Wang, S. H. (2008). Self-deployment by density control in sensor networks. IEEE Transactions on Vehicular Technology, 57(3), 1745-1755.

[7] Jae-Hyun, S. E. O., Yong-Hyuk, K. I. M., Hwang-Bin, R. Y. O. U., Si-Ho, C. H. A., \& Minho, J. O. (2008). Optimal sensor deployment for wireless surveillance sensor networks by a hybrid steady-state genetic algorithm. IEICE Transactions on Communications, 91(11), 3534-3543.

[8] Aziz, N. A. A., Aziz, K. A., \& Ismail, W. Z. W. (2009). Coverage strategies for wireless sensor networks. World Academy of Science, Engineering and Technology, 50, 145-150. 
[9] Shen, X., Chen, J., \& Sun, Y. (2006). Grid scan: A simple and effective approach for coverage issue in wireless sensor networks. Proceedings of the IEEE International Conference on Communications: Vol. 8. (pp. 3480-3484). NJ: IEEE.

[10] Bai, X., Kumar, S., Xuan, D., Yun, Z., \& Lai, T. H. (2006). Deploying wireless sensors to achieve both coverage and connectivity. Proceedings of the $7^{\text {th }}$ ACM International Symposium on Mobile ad Hoc Networking and Computing (pp. 131-142). ACM.

[11] Mahboubi, H., Moezzi, K., Aghdam, A. G., Sayrafian-Pour, K., \& Marbukh, V. (2011). Distributed deployment algorithms for improved coverage in mobile sensor networks. Proceedings of the IEEE International Conference on Control Applications (CCA) (pp. 1244-1249). NJ: IEEE.

[12] Mulligan, R., \& Ammari, H. M. (2010). Coverage in wireless sensor networks: A survey. Network Protocols and Algorithms, 2(2), 27-53.

[13] Norouzi, A., \& Zaim, A. H. (2014). Genetic algorithm application in optimization of wireless sensor networks. The Scientific World Journal.

[14] Qu, Y., \& Georgakopoulos, S. V. (2011). Relocation of wireless sensor network nodes using a genetic algorithm. Proceedings of the $12^{\text {th }}$ IEEE Annual International Conference on Wireless and Microwave Technology (WAMICON) (pp. 1-5). NJ: IEEE.

[15] Bhondekar, A. P., Vig, R., Singla, M. L., Ghanshyam, C., \& Kapur, P. (2009). Genetic algorithm based node placement methodology for wireless sensor networks. Proceedings of the International Multiconference of Engineers and Computer Scientists: Vol. 1 (pp. 18-20).

[16] Fei, X., Samarah, S., \& Boukerche, A. (2010). A bio-inspired coverage-aware scheduling scheme for wireless sensor networks. Proceedings of the IEEE International Symposium on Parallel \& Distributed Processing, Workshops and PHD Forum (IPDPSW) (pp. 1-8). NJ: IEEE.

[17] Yoon, Y., \& Kim, Y. H. (2013). An efficient genetic algorithm for maximum coverage deployment in wireless sensor networks. IEEE Transactions on Cybernetics, 43(5), 1473-1483.

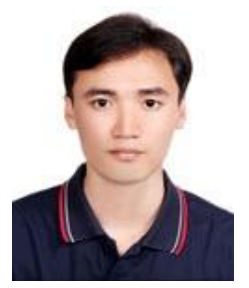

Ta Van Dai received the B.S. degree in computer science and engineering from Vietnam National University of Agriculture, Vietnam, in 2012. Now, he is currently a graduate student of National Formosa University, Taiwan. His current research interests are networking, network security, wireless sensor networks.

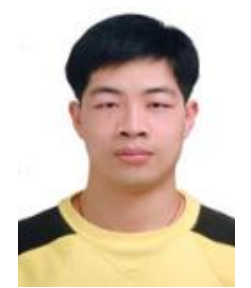

Shih-Chang Huang received the Ph.D. degree in computer and information science at National Chiao-Tung University, Taiwan, in 2008. He joined the Department of Computer Science and Information Engineering, National Formosa University, in 2009. Dr. Huang is currently an associate professor. His research interests include wireless local area networks, wireless sensor networks, and smart home. He is an IEEE member.

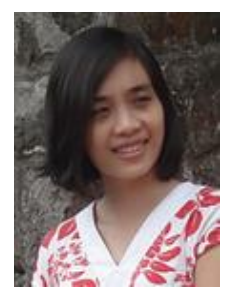

Huynh Thi Thanh Binh is working at the School of Information and Communication Technology, Hanoi University of Science and Technology. Her research interests include computational intelligence: genetic algorithm. She is the treasurer of IEEE Viet Nam Section, chair of IEEE Computational Intelligence Section, and the chair of IEEE Young Professional Chapter. She is currently an associate professor. She has served as the organizing chair of several conferences and is a program committee member of over 20 international conferences and journals. 